Research Article

\title{
Maximum Reciprocal Degree Resistance Distance Index of Bicyclic Graphs
}

\author{
Gaixiang Cai $\mathbb{D},{ }^{1}$ Xing-Xing $\mathrm{Li}^{1},{ }^{1}$ and Guidong Yu $\mathbb{D}{ }^{1,2}$ \\ ${ }^{1}$ School of Mathematics and Physics, Anqing Normal University, Anqing 246133, China \\ ${ }^{2}$ Basic Department, Hefei Preschool Education College, Hefei 230013, China \\ Correspondence should be addressed to Guidong Yu; guidongy@163.com
}

Received 22 March 2021; Accepted 7 June 2021; Published 19 June 2021

Academic Editor: Junwei Wang

Copyright (c) 2021 Gaixiang Cai et al. This is an open access article distributed under the Creative Commons Attribution License, which permits unrestricted use, distribution, and reproduction in any medium, provided the original work is properly cited.

The reciprocal degree resistance distance index of a connected graph $G$ is defined as $\operatorname{RDR}(G)=\sum_{\{u, v\} \subseteq V(G)}\left(\left(\mathrm{d}_{G}(u)+\right.\right.$ $\left.\left.\mathrm{d}_{G}(v)\right) / r_{G}(u, v)\right)$, where $r_{G}(u, v)$ is the resistance distance between vertices $u$ and $v$ in $G$. Let $\mathscr{B}_{n}$ denote the set of bicyclic graphs without common edges and with $n$ vertices. We study the graph with the maximum reciprocal degree resistance distance index among all graphs in $\mathscr{B}_{n}$ and characterize the corresponding extremal graph.

\section{Introduction}

Let $G=(V(G), E(G))$ be a simple connected graph of order $n$ with vertex set $V(G)=\left\{v_{1}, v_{2}, \ldots, v_{n}\right\}$ and edge set $E(G)$. For any vertex, $u \in V(G)$, denoted by $\mathrm{d}_{G}(u)$ the degree of vertex $u$. The distance between vertices of $u$ and $v$ is defined as the length of the shortest path between them and denoted by $\mathrm{d}_{G}(u, v)$. The resistance distance between vertices of $u$ and $v$, denoted by $r_{G}(u, v)$, is the effective resistance between the two nodes of the electronic network obtained so that its nodes correspond to the vertices of $G$ and each edge of $G$ is replaced by a resistor of unit resistance.

Topological indices are numbers related to molecular structures, used as quantitative relationships between chemical structures and properties. Some of them are based on the distance of graph [1], the vertex degree [2], or the resistance distance. The first topological index was published by Wiener [3]. From then, many topological indices are defined, such as the Harary index [4], Kirchhoff index $[5,6]$, the first and the second Zagreb indices $[7,8]$, and degree distance $[9,10]$.

Recently, Alizadeh and Iranmanesh [11] proposed a new topological index, the reciprocal degree distance, which is defined as

$$
\operatorname{RDD}(G)=\frac{1}{2} \sum_{\{u, v\} \in V(G)} \frac{\mathrm{d}_{G}(u)+\mathrm{d}_{G}(v)}{\mathrm{d}_{G}(u, v)}
$$

Its lower and upper bounds were obtained in [12], and its chemical applications and mathematical properties have been well studied in [11, 13].

Especially, analogous to the reciprocal degree distance index $\operatorname{RDD}(G)$, Cai et al. [14] introduced a new graph invariant based on both the vertex degree and the resistance, named the reciprocal degree resistance distance index, shown as follows:

$$
\operatorname{RDR}(G)=\sum_{\{u, v\} \subseteq V(G)} \frac{\mathrm{d}_{G}(u)+\mathrm{d}_{G}(v)}{r_{G}(u, v)} .
$$

A bicyclic (or unicyclic) graph is a connected graph of order $n$ with $n+1$ (or $n$ ) edges. Let $\mathscr{B}_{n}$ be the set of bicyclic graphs without common edges of order $n$. Tree, unicyclic graph, and bicyclic graph are three kinds of graphs with a simple structure; the reciprocal degree resistance distance index of tree is the same as the reciprocal degree distance, Cai et al. [14] determined the graph with the maximum reciprocal degree resistance distance index among all unicyclic graph and characterized the corresponding extremal 
graph. In this paper, we determine the graph with the maximum reciprocal degree resistance distance index among all graphs in $\mathscr{B}_{n}$ and characterize the corresponding extremal graph.

This paper is organized as follows: in the second part, we give three types of transformation, edge-lifting transformation, cycle-lifting transformation, and cycle-shrinking transformation, to keep the reciprocal degree resistance distance index increasing. In the third part, we give the maximum reciprocal degree resistance distance index among all graphs in $\mathscr{B}_{n}$.

\section{Preliminaries}

Let $C_{g}=v_{1} v_{2}, \ldots, v_{g} v_{1}$ be the cycle graph with girth $g \geq 3$. For any two vertices $v_{i}, v_{j} \in V\left(C_{g}\right)$ with $i<j$, by Ohm's law, one has

$$
r_{C_{g}}\left(v_{i}, v_{j}\right)=\frac{(j-i)(g+i-j)}{g} .
$$

Lemma 1 (See [6]). Let $x$ be a cut vertex of a connected graph $G$ and $s$ and $t$ be two vertices occurring in different components which arise upon the deletion of $x$.Then,

$$
r_{G}(s, t)=r_{G}(s, x)+r_{G}(x, t) .
$$

2.1. Edge-Lifting Transformation. Let $G^{\prime}$ be obtained from $G$ by an edge-lifting transformation at $e=\left\{u_{0}, v_{0}\right\}$, see Figure 1 . See [14] for specific description.

Lemma 2 (See [14]). If $G^{\prime}$ can be obtained from $G$ by an edge-lifting transformation, see Figure 1, then $R D R(G)<R D R\left(G^{\prime}\right)$.

2.2. Cycle-Lifting Transformation. Let $G_{p}^{i}$ be the star graph $K_{1, s_{i}}(1 \leq i \leq p)$ with order $s_{i}+1$ and $G_{q}^{j}$ be the star graph $K_{1, t_{j}}(2 \leq j \leq q)$ with order $t_{j}+1$. Let $\mathscr{C}$ be a bicyclic graph with exactly two cycles, which are $C_{p}=v_{1} v_{2}, \ldots, v_{p} v_{1}$ and $C_{q}=v_{1} u_{2}, \ldots, u_{q} v_{1}\left(v_{i} \neq u_{j}, 1 \leq i \leq p, 2 \leq j \leq q\right)$, and $G$ is obtained from $\mathscr{C}$ by identifying $v_{i}$ with the center of $G_{p}^{i}$ for all $i(1 \leq i \leq p)$ and $u_{j}$ with the center of $G_{q}^{j}$ for all $j(2 \leq j \leq q)$. Deleting all edges in $G_{p}^{i}(2 \leq i \leq p)$, joining $v_{1}$ to all pendent vertices of $G_{p}^{i}(2 \leq i \leq p)$, we obtained a new graph, denoted by $G^{\prime}$, see Figure 2 . This operation is called a cycle-lifting transformation of $G$ with respect to $C_{p}$.

Lemma 3. If $G^{\prime}$ can be obtained from $G$ by a cycle-lifting transformation, see Figure 2, and $\sum_{i=2}^{p} s_{i}>0$, then $R D R(G)<R D R\left(G^{\prime}\right)$.

Proof. Let $G$ and $G^{\prime}$ be as shown in Figure 2. By the definition of $\operatorname{RDR}(G)$,

$$
\begin{aligned}
& \operatorname{RDR}(G)=\sum_{i=1}^{p} \sum_{x, y \in V\left(G_{p}^{i}\right) \backslash\left\{v_{i}\right\}} \frac{\mathrm{d}_{G}(x)+\mathrm{d}_{G}(y)}{r_{G}(x, y)}+\sum_{i=1}^{p} \sum_{x \in V\left(G_{p}^{i}\right) \backslash\left\{v_{i}\right\}} \frac{\mathrm{d}_{G}\left(v_{i}\right)+\mathrm{d}_{G}(x)}{r_{G}\left(v_{i}, x\right)}+\sum_{i=1}^{p} \sum_{x \in V\left(G_{p}^{i}\right) \backslash\left\{v_{i}\right\} y \in V\left(C_{p}\right) \backslash\left\{v_{i}\right\}} \frac{\mathrm{d}_{G}(x)+\mathrm{d}_{G}(y)}{r_{G}(x, y)} \\
& +\sum_{i, j=1 i \neq j}^{p} \sum_{x \in V\left(G_{p}^{i}\right) \backslash\left\{v_{i}\right\} y \in V\left(G_{p}^{j}\right) \backslash\left\{v_{j}\right\}} \frac{\mathrm{d}_{G}(x)+\mathrm{d}_{G}(y)}{r_{G}(x, y)}+\sum_{i=1}^{p} \sum_{x \in V\left(G_{p}^{i}\right) \backslash\left\{v_{i}\right\} y \in V\left(C_{q}\right) \backslash\left\{v_{1}\right\}} \frac{\mathrm{d}_{G}(x)+\mathrm{d}_{G}(y)}{r_{G}(x, y)} \\
& +\sum_{i=1 x \in V\left(G_{p}^{i}\right) \backslash\left\{v_{i}\right\}}^{p} \sum_{j=2 y \in V\left(G_{q}^{j}\right) \backslash\left\{u_{j}\right\}}^{q} \frac{\mathrm{d}_{G}(x)+\mathrm{d}_{G}(y)}{r_{G}(x, y)} \\
& +\sum_{x, y \in V\left(C_{p}\right)} \frac{\mathrm{d}_{G}(x)+\mathrm{d}_{G}(y)}{r_{G}(x, y)}+\sum_{x \in V\left(C_{p}\right)} \sum_{y \in V\left(C_{q}\right) \backslash\left\{v_{1}\right\}} \frac{\mathrm{d}_{G}(x)+\mathrm{d}_{G}(y)}{r_{G}(x, y)}+\sum_{j=2}^{q} \sum_{x \in V\left(C_{p}\right)} \sum_{y \in V\left(G_{p}^{j}\right) \backslash\left\{u_{j}\right\}} \frac{\mathrm{d}_{G}(x)+\mathrm{d}_{G}(y)}{r_{G}(x, y)} \\
& +\sum_{x, y \in V\left(C_{q}\right) \backslash\left\{v_{1}\right\}} \frac{\mathrm{d}_{G}(x)+\mathrm{d}_{G}(y)}{r_{G}(x, y)} \\
& +\sum_{j=2}^{q} \sum_{x \in V\left(C_{q}\right) \backslash\left\{v_{1}\right\} y \in V\left(G_{p}^{j}\right) \backslash\left\{u_{j}\right\}} \frac{\mathrm{d}_{G}(x)+\mathrm{d}_{G}(y)}{r_{G}(x, y)}+\sum_{j=2}^{q} \sum_{x, y \in V\left(G_{p}^{j}\right) \backslash\left\{u_{j}\right\}} \frac{\mathrm{d}_{G}(x)+\mathrm{d}_{G}(y)}{r_{G}(x, y)} \\
& +\sum_{i, j=2 i \neq j}^{q} \sum_{x \in V\left(G_{q}^{i}\right) \backslash\left\{u_{i}\right\} y \in V\left(G_{q}^{j}\right) \backslash\left\{u_{j}\right\}} \frac{\mathrm{d}_{G}(x)+\mathrm{d}_{G}(y)}{r_{G}(x, y)} .
\end{aligned}
$$




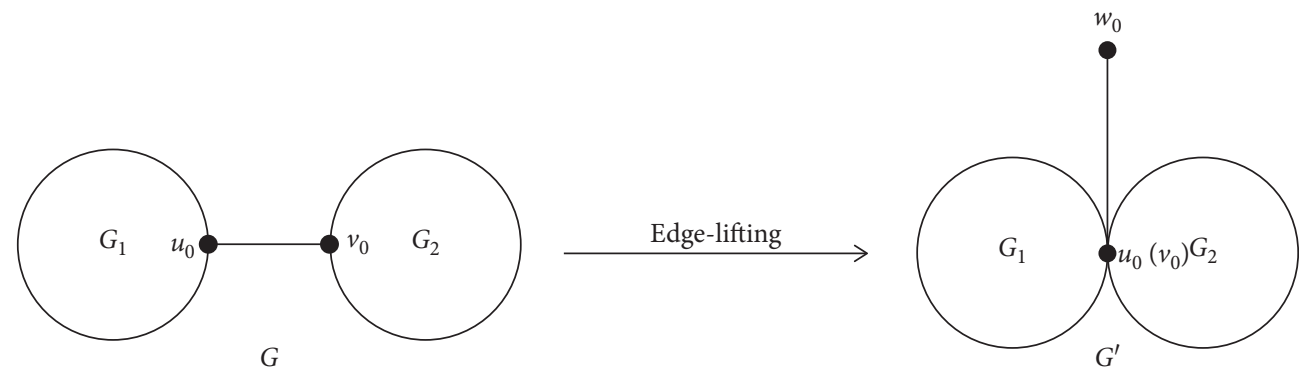

Figure 1: The edge-lifting transformation.
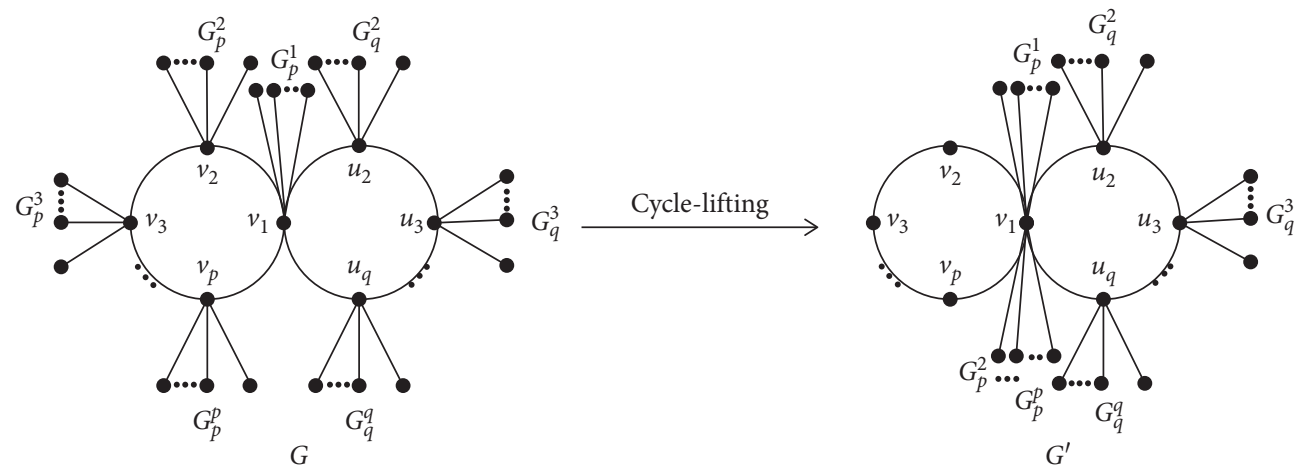

Figure 2: The cycle-lifting transformation.

$$
\begin{aligned}
& \operatorname{RDR}\left(G^{\prime}\right)=\sum_{i=1}^{p} \sum_{x, y \in V\left(G_{p}^{i}\right) \backslash\left\{v_{1}\right\}} \frac{\mathrm{d}_{G^{\prime}}(x)+\mathrm{d}_{G^{\prime}}(y)}{r_{G^{\prime}}(x, y)}+\sum_{i=1}^{p} \sum_{x \in V\left(G_{p}^{i}\right) \backslash\left\{v_{1}\right\}} \frac{\mathrm{d}_{G^{\prime}}(x)+\mathrm{d}_{G^{\prime}}(y)}{r_{G^{\prime}}(x, y)}+\sum_{i=1}^{p} \sum_{x \in V\left(G_{p}^{i}\right) \backslash\left\{v_{1}\right\} y \in V\left(C_{p}\right) \backslash\left\{v_{1}\right\}} \frac{\mathrm{d}_{G^{\prime}}(x)+\mathrm{d}_{G^{\prime}}(y)}{r_{G^{\prime}}(x, y)} \\
& +\sum_{i, j=1 i \neq j}^{p} \sum_{x \in V\left(G_{p}^{i}\right) \backslash\left\{v_{1}\right\} y \in V} \frac{\mathrm{d}_{G^{\prime}}(x)+\mathrm{d}_{G^{\prime}}(y)}{r_{G^{\prime}}(x, y)}+\sum_{i=1}^{p} \sum_{x \in V\left(G_{p}^{i}\right) \backslash\left\{v_{1}\right\}} \sum_{y \in V\left(c_{q}\right) \backslash\left\{v_{1}\right\}} \frac{\mathrm{d}_{G^{\prime}}(x)+\mathrm{d}_{G^{\prime}}(y)}{r_{G^{\prime}}(x, y)} \\
& +\sum_{i=1 x \in V\left(G_{p}^{i}\right) \backslash\left\{v_{1}\right\}}^{p} \sum_{j=2 y \in V\left(G_{q}^{j}\right) \backslash\left\{u_{j}\right\}}^{q} \frac{\mathrm{d}_{G^{\prime}}(x)+\mathrm{d}_{G^{\prime}}(y)}{r_{G^{\prime}}(x, y)}
\end{aligned}
$$

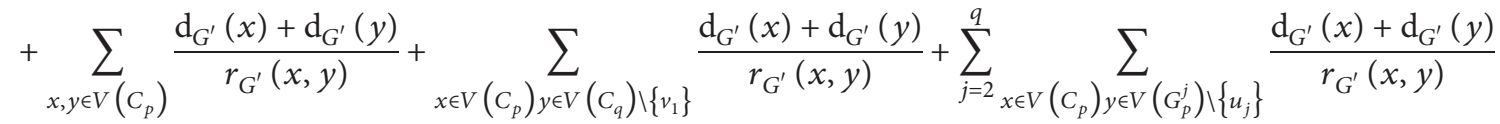

$$
\begin{aligned}
& +\sum_{x, y \in V\left(C_{q}\right) \backslash\left\{v_{1}\right\}} \frac{\mathrm{d}_{G^{\prime}}(x)+\mathrm{d}_{G^{\prime}}(y)}{r_{G^{\prime}}(x, y)} \\
& +\sum_{j=2}^{q} \sum_{x \in V\left(C_{q}\right) \backslash\left\{v_{1}\right\} y \in V\left(G_{p}^{j}\right) \backslash\left\{u_{j}\right\}} \frac{\mathrm{d}_{G^{\prime}}(x)+\mathrm{d}_{G^{\prime}}(y)}{r_{G^{\prime}}(x, y)}+\sum_{j=2}^{q} \sum_{x, y \in V\left(G_{p}^{j}\right) \backslash\left\{u_{j}\right\}} \frac{\mathrm{d}_{G^{\prime}}(x)+\mathrm{d}_{G^{\prime}}(y)}{r_{G^{\prime}}(x, y)} \\
& +\sum_{i, j=2 i \neq j}^{q} \sum_{x \in V\left(G_{q}^{i}\right) \backslash\left\{u_{i}\right\} y \in V\left(G_{q}^{j}\right) \backslash\left\{u_{j}\right\}} \frac{\mathrm{d}_{G^{\prime}}(x)+\mathrm{d}_{G^{\prime}}(y)}{r_{G^{\prime}}(x, y)} .
\end{aligned}
$$

(1) Noting that $\mathrm{d}_{G}(x)+\mathrm{d}_{G}(y)=\mathrm{d}_{G^{\prime}}(x)+\mathrm{d}_{G^{\prime}}(y)$, $r_{G}(x, y)=r_{G^{\prime}}(x, y)$ for $x, y \in V\left(G_{p}^{i}\right) \backslash\left\{v_{i}\right\}$ of $G$ and $x, y \in V\left(G_{p}^{i}\right) \backslash\left\{v_{1}\right\}$ of $G^{\prime}$, we have 


$$
\sum_{i=1}^{p} \sum_{x, y \in V\left(G_{p}^{i}\right) \backslash\left\{v_{1}\right\}} \frac{\mathrm{d}_{G^{\prime}}(x)+\mathrm{d}_{G^{\prime}}(y)}{r_{G^{\prime}}(x, y)}-\sum_{i=1}^{p} \sum_{x, y \in V\left(G_{p}^{i}\right) \backslash\left\{v_{i}\right\}} \frac{\mathrm{d}_{G}(x)+\mathrm{d}_{G}(y)}{r_{G}(x, y)}=0 .
$$

(2) Noting that $r_{G}\left(v_{i}, x\right)=r_{G^{\prime}}\left(v_{1}, x\right)=1, \quad \mathrm{~d}_{G}\left(v_{1}\right)+$ $\mathrm{d}_{G}(x)=s_{1}+5$ for $x \in V\left(G_{p}^{1}\right) \backslash\left\{v_{1}\right\}$ and $\mathrm{d}_{G}\left(v_{i}\right)+$ $\mathrm{d}_{G}(x)=s_{i}+3$ for $x \in V\left(G_{p}^{i}\right) \backslash\left\{v_{i}\right\}(2 \leq i \leq p)$ of $G$, and

$\mathrm{d}_{G^{\prime}}\left(v_{1}\right)+\mathrm{d}_{G^{\prime}}(x)=s_{1}+\cdots+s_{p}+5$ for $x \in V\left(G_{p}^{i}\right) \backslash\left\{v_{1}\right\}$ of $G^{\prime}$, we have

$$
\sum_{i=1}^{p} \sum_{x \in V} \frac{\mathrm{d}_{G^{\prime}}\left(v_{1}\right)+\mathrm{d}_{G^{\prime}}(x)}{r_{G^{\prime}}\left(v_{1}, y\right)}-\sum_{i=1}^{p} \sum_{x \in V} \sum_{\left(G_{p}^{i}\right) \backslash\left\{v_{i}\right\}} \frac{\mathrm{d}_{G}\left(v_{i}\right)+\mathrm{d}_{G}(x)}{r_{G}\left(v_{i}, y\right)}=\sum_{i, j=1 i \neq j}^{p} s_{i} \cdot s_{j}+2 \sum_{i=2}^{p} s_{i} .
$$

(3) Noting that $\mathrm{d}_{G}(x)+\mathrm{d}_{G}\left(v_{1}\right)=s_{1}+5, r_{G}\left(x, v_{1}\right)=1+$ $r_{G}\left(v_{i}, v_{1}\right)$ for $x \in V\left(G_{p}^{i}\right) \backslash\left\{v_{i}\right\}(2 \leq i \leq p)$ of $G$, $\mathrm{d}_{G}(x)+\mathrm{d}_{G}(y)=s_{j}+3, r_{G}(x, y)=1+r_{G}\left(v_{i}, v_{j}\right)$, for $x \in V\left(G_{p}^{i}\right) \backslash\left\{v_{i}\right\}(1 \leq i \leq p), y=v_{j} \in V\left(C_{p}\right) \backslash\left\{v_{1}, v_{i}\right\}$ of
$G, \quad$ and $\quad \mathrm{d}_{G^{\prime}}(x)+\mathrm{d}_{G^{\prime}}(y)=3, \quad r_{G^{\prime}}(x, y)=1+$ $r_{G^{\prime}}\left(v_{1}, y\right)$ for $x \in V\left(G_{p}^{i}\right) \backslash\left\{v_{1}\right\}, y \in V\left(C_{p}\right) \backslash\left\{v_{1}\right\}$ of $G^{\prime}$, we have

$$
\begin{aligned}
& \sum_{i=1}^{p} \sum_{x \in V\left(G_{p}^{i}\right) \backslash\left\{v_{1}\right\} y \in V\left(C_{p}\right) \backslash\left\{v_{1}\right\}} \frac{\mathrm{d}_{G^{\prime}}(x)+\mathrm{d}_{G^{\prime}}(y)}{r_{G^{\prime}}(x, y)}-\sum_{i=1}^{p} \sum_{x \in V} \sum_{\left(G_{p}^{i}\right) \backslash\left\{v_{i}\right\} y \in V\left(C_{p}\right) \backslash\left\{v_{i}\right\}} \frac{\mathrm{d}_{G}(x)+\mathrm{d}_{G}(y)}{r_{G}(x, y)} \\
& =-\left(\sum_{i, j=1 i \neq j}^{p} \frac{s_{i} \cdot s_{j}}{1+r_{G}\left(v_{i}, v_{j}\right)}+2 \sum_{i=2}^{p} \frac{s_{i}}{1+r_{G}\left(v_{i}, v_{1}\right)}\right) .
\end{aligned}
$$

(4) Noting that $\mathrm{d}_{G}(x)+\mathrm{d}_{G}(y)=\mathrm{d}_{G^{\prime}}(x)+\mathrm{d}_{G^{\prime}}(y)$, $r_{G}(x, y)=r_{G}\left(x, v_{i}\right)+r_{G}\left(v_{i}, v_{j}\right)+r_{G}\left(v_{j}, y\right)>r_{G^{\prime}}(x$, $\left.v_{1}\right)+r_{G^{\prime}}\left(v_{1}, y\right)=r_{G^{\prime}}(x, y) \quad$ for $\quad x \in V\left(G_{p}^{i}\right) \backslash\left\{v_{i}\right\}$, $y \in V\left(G_{p}^{j}\right) \backslash\left\{v_{j}\right\}(1 \leq i, j \leq p, i \neq j)$ of $G$ and $x \in$ $V\left(G_{p}^{i}\right) \backslash\left\{v_{1}\right\}, y \in V\left(G_{p}^{j}\right) \backslash\left\{v_{1}\right\}(1 \leq i, j \leq p, i \neq j)$ of $G^{\prime}$, similarly for $\quad x \in V\left(G_{p}^{i}\right) \backslash\left\{v_{i}\right\}(1 \leq i \leq p), y \in$ $V\left(C_{q}\right) \backslash\left\{v_{1}\right\} \quad$ and $\quad x \in V\left(G_{p}^{i}\right) \backslash\left\{v_{1}\right\}(1 \leq i \leq p), y \in$
$V\left(C_{q}\right) \backslash\left\{v_{1}\right\}$ of $G$ and $G^{\prime}$, respectively, and for $x \in V\left(G_{p}^{i}\right) \backslash\left\{v_{i}\right\} \quad(1 \leq i \leq p), y \in V\left(G_{q}^{j}\right) \backslash\left\{u_{j}\right\}$ $(2 \leq j \leq q) \quad$ and $\quad x \in V\left(G_{p}^{i}\right) \backslash\left\{v_{1}\right\} \quad(1 \leq i \leq p)$, $y \in V\left(G_{q}^{j}\right) \backslash\left\{u_{j}\right\}(2 \leq j \leq q)$ of $G$ and $G^{\prime}$, respectively, we have the same conclusions; thus,

$$
\begin{aligned}
& \left(\sum_{i, j=1 i \neq j}^{p} \sum_{x \in V\left(G_{p}^{i}\right) \backslash\left\{v_{1}\right\} y \in V\left(G_{p}^{j}\right) \backslash\left\{v_{1}\right\}} \frac{\mathrm{d}_{G^{\prime}}(x)+\mathrm{d}_{G^{\prime}}(y)}{r_{G^{\prime}}(x, y)}-\sum_{i, j=1 i \neq j}^{p} \sum_{x \in V\left(G_{p}^{i}\right) \backslash\left\{v_{i}\right\} y \in V\left(G_{p}^{j}\right) \backslash\left\{v_{j}\right\}} \frac{\mathrm{d}_{G}(x)+\mathrm{d}_{G}(y)}{r_{G}(x, y)}\right) \\
& +\left(\sum_{i=1}^{p} \sum_{x \in V\left(G_{p}^{i}\right) \backslash\left\{v_{1}\right\} y \in V\left(C_{q}\right) \backslash\left\{v_{1}\right\}} \frac{\mathrm{d}_{G^{\prime}}(x)+\mathrm{d}_{G^{\prime}}(y)}{r_{G^{\prime}}(x, y)}-\sum_{i=1}^{p} \sum_{x \in V\left(G_{p}^{i}\right) \backslash\left\{v_{i}\right\} y \in V\left(C_{q}\right) \backslash\left\{v_{1}\right\}} \frac{\mathrm{d}_{G}(x)+\mathrm{d}_{G}(y)}{r_{G}(x, y)}\right) \\
& +\left(\sum_{i=1 x \in V\left(G_{p}^{i}\right) \backslash\left\{v_{1}\right\}}^{p} \sum_{j=2 y \in V\left(G_{q}^{j}\right) \backslash\left\{u_{j}\right\}}^{q} \frac{\mathrm{d}_{G^{\prime}}(x)+\mathrm{d}_{G^{\prime}}(y)}{r_{G^{\prime}}(x, y)}-\sum_{i=1 x \in V\left(G_{p}^{i}\right) \backslash\left\{v_{i}\right\}}^{p} \sum_{j=2 y \in V\left(G_{q}^{j}\right) \backslash\left\{u_{j}\right\}}^{q} \frac{\mathrm{d}_{G}(x)+\mathrm{d}_{G}(y)}{r_{G}(x, y)}\right)>0
\end{aligned}
$$

presents. 
(5) We note that $\mathrm{d}_{G}\left(v_{1}\right)=s_{1}+4, \mathrm{~d}_{G}\left(v_{i}\right)=s_{i}+2(2 \leq$ $i \leq p)$ for any $v_{i} \in V\left(C_{p}\right)$ of $G$ and $\mathrm{d}_{G^{\prime}}\left(v_{1}\right)=s_{1}+\cdots+s_{p}+4, \mathrm{~d}_{G^{\prime}}\left(v_{i}\right)=2(2 \leq i \leq p)$ for any $v_{i} \in V\left(C_{p}\right)$ of $G^{\prime}$. Let $r_{G}\left(v_{i}, v_{j}\right)=r_{G^{\prime}}\left(v_{i}, v_{j}\right)=r_{t}$, when $|j-i|=t$; then, $r_{t}=r_{p-t}$. Thus, we have

$$
\sum_{x, y \in V\left(C_{p}\right)} \frac{\mathrm{d}_{G}(x)+\mathrm{d}_{G}(y)}{r_{G}(x, y)}=\sum_{i=2}^{p} \frac{\mathrm{d}_{G}\left(v_{1}\right)+\mathrm{d}_{G}\left(v_{i}\right)}{r_{G}\left(v_{1}, v_{i}\right)}+\sum_{i=3}^{p} \frac{\mathrm{d}_{G}\left(v_{2}\right)+\mathrm{d}_{G}\left(v_{i}\right)}{r_{G}\left(v_{2}, v_{i}\right)}+\cdots+\frac{\mathrm{d}_{G}\left(v_{p-1}\right)+\mathrm{d}_{G}\left(v_{p}\right)}{r_{G}\left(v_{p-1}, v_{p}\right)}
$$

and then, we have

$$
\begin{aligned}
& \sum_{i=2}^{p} \frac{\mathrm{d}_{G}\left(v_{1}\right)+\mathrm{d}_{G}\left(v_{i}\right)}{r_{G}\left(v_{1}, v_{i}\right)}=\frac{s_{1}+s_{2}+6}{r_{1}}+\frac{s_{1}+s_{3}+6}{r_{2}}+\cdots+\frac{s_{1}+s_{p}+6}{r_{p-1}}, \\
& \sum_{i=3}^{p} \frac{\mathrm{d}_{G}\left(v_{2}\right)+\mathrm{d}_{G}\left(v_{i}\right)}{r_{G}\left(v_{2}, v_{i}\right)}=\frac{s_{2}+s_{3}+4}{r_{1}}+\cdots+\frac{s_{2}+s_{p}+4}{r_{p-2}}, \\
& \cdots \\
& \frac{\mathrm{d}_{G}\left(v_{p-1}\right)+\mathrm{d}_{G}\left(v_{p}\right)}{r_{G}\left(v_{p-1}, v_{p}\right)}=\frac{s_{p-1}+s_{p}+4}{r_{1}} .
\end{aligned}
$$

Thus,

$$
\begin{gathered}
\sum_{x, y \in V\left(c_{p}\right)} \frac{\mathrm{d}_{G}(x)+\mathrm{d}_{G}(y)}{r_{G}(x, y)}=\frac{\left(s_{1}+\cdots+s_{p-1}\right)+\left(s_{2}+\cdots+s_{p}\right)+4(p-2)+6}{r_{1}} \\
+\frac{\left(s_{1}+\cdots+s_{p-2}\right)+\left(s_{3}+\cdots+s_{p}\right)+4(p-3)+6}{r_{2}}+\cdots+\frac{s_{1}+s_{p}+6}{r_{p-1}} .
\end{gathered}
$$

Similarly,

$\sum_{x, y \in V\left(C_{p}\right)} \frac{\mathrm{d}_{G^{\prime}}(x)+\mathrm{d}_{G^{\prime}}(y)}{r_{G^{\prime}}(x, y)}=\frac{\left(s_{1}+\cdots+s_{p}\right)+4(p-2)+6}{r_{1}}+\frac{\left(s_{1}+\cdots+s_{p}\right)+4(p-3)+6}{r_{2}}+\cdots+\frac{s_{1}+\cdots+s_{p}+6}{r_{p-1}}$.

Then,

$\sum_{x, y \in V\left(C_{p}\right)} \frac{\mathrm{d}_{G^{\prime}}(x)+\mathrm{d}_{G^{\prime}}(y)}{r_{G^{\prime}}(x, y)}-\sum_{x, y \in V\left(C_{p}\right)} \frac{\mathrm{d}_{G}(x)+\mathrm{d}_{G}(y)}{r_{G}(x, y)}=\frac{s_{2}+\cdots+s_{p-1}}{r_{1}}+\frac{s_{3}+\cdots+s_{p-2}}{r_{2}}+\cdots+\frac{-\left(s_{2}+\cdots+s_{p-1}\right)}{r_{p-1}}=0$. 
(6) It is noted that $\mathrm{d}_{G}\left(v_{1}\right)=s_{1}+4, \quad \mathrm{~d}_{G}\left(v_{i}\right)=s_{i}+$ $2(2 \leq i \leq p), \quad \mathrm{d}_{G}\left(u_{j}\right)=t_{j}+2(2 \leq j \leq q) \quad$ for $\quad v_{i} \in$ $V\left(C_{p}\right)$ and $u_{j} \in V\left(C_{q}\right) \backslash\left\{v_{1}\right\}$ of $G$. Similarly, It is noted that $\mathrm{d}_{G^{\prime}}\left(v_{1}\right)=\sum_{i=1}^{p} s_{i}+4, \quad \mathrm{~d}_{G^{\prime}}\left(v_{i}\right)=2(2 \leq$ $i \leq p), \mathrm{d}_{G^{\prime}}\left(u_{j}\right)=t_{j}+2(2 \leq i \leq q)$, for $v_{i} \in V\left(C_{p}\right)$ and $u_{j} \in V\left(C_{q}\right) \backslash\left\{v_{1}\right\}$ of $G^{\prime}$. Let $r_{G}\left(v_{i}, v_{1}\right)=r_{p}^{t}$, when $i-1=t(2 \leq i \leq p, 1 \leq t \leq p-1)$. Let $r_{G}\left(v_{1}, u_{j}\right)=r_{q}^{s}$, when $j-1=s(2 \leq j \leq q, 1 \leq s \leq q-1)$. Then, $r_{G}(x, y)=$ $r_{G}\left(v_{i}, v_{1}\right)+r_{G}\left(v_{1}, u_{i}\right)=r_{p}^{t}+r_{q}^{s}=r_{G^{\prime}}(x, y)$

$x \in V\left(C_{p}\right)$ and $y \in V\left(C_{q}\right) \backslash\left\{v_{1}\right\}$. Thus, we have

$$
\sum_{x \in V\left(c_{p}\right)} \frac{\mathrm{d}_{G}(x)+\mathrm{d}_{G}(y)}{r_{G}(x, y)}=\sum_{i=2}^{q} \frac{\mathrm{d}_{G}\left(v_{1}\right)+\left\{v_{1}\right\}}{r_{G}\left(v_{1}, u_{i}\right)}+\sum_{i=2}^{q} \frac{\mathrm{d}_{G}\left(v_{2}\right)+\mathrm{d}_{G}\left(u_{i}\right)}{r_{G}\left(v_{2}, u_{i}\right)}+\cdots+\sum_{i=2}^{q} \frac{\mathrm{d}_{G}\left(v_{p}\right)+\mathrm{d}_{G}\left(u_{i}\right)}{r_{G}\left(v_{p}, u_{i}\right)},
$$

and then, we have

$$
\begin{gathered}
\sum_{i=2}^{q} \frac{\mathrm{d}_{G}\left(v_{1}\right)+\mathrm{d}_{G}\left(u_{i}\right)}{r_{G}\left(v_{1}, u_{i}\right)}=\frac{s_{1}+t_{2}+6}{r_{q}^{1}}+\frac{s_{1}+t_{3}+6}{r_{q}^{2}}+\cdots+\frac{s_{1}+t_{q}+6}{r_{q}^{q-1}}, \\
\sum_{i=2}^{q} \frac{\mathrm{d}_{G}\left(v_{2}\right)+\mathrm{d}_{G}\left(u_{i}\right)}{r_{G}\left(v_{2}, u_{i}\right)}=\frac{s_{2}+t_{2}+4}{r_{p}^{1}+r_{q}^{1}}+\frac{s_{2}+t_{3}+4}{r_{p}^{1}+r_{q}^{2}}+\cdots+\frac{s_{2}+t_{q}+4}{r_{p}^{1}+r_{q}^{q-1}}, \\
\ldots \\
\sum_{i=2}^{q} \frac{\mathrm{d}_{G}\left(v_{p}\right)+\mathrm{d}_{G}\left(u_{i}\right)}{r_{G}\left(v_{p}, u_{i}\right)}=\frac{s_{p}+t_{2}+4}{r_{p}^{p-1}+r_{q}^{1}}+\frac{s_{p}+t_{3}+4}{r_{p}^{p-1}+r_{q}^{2}}+\cdots+\frac{s_{p}+t_{q}+4}{r_{p}^{p-1}+r_{q}^{q-1}} .
\end{gathered}
$$

Similarly,

$\sum_{x \in V\left(C_{p}\right) y_{y \in V}\left(C_{q}\right) \backslash\left\{v_{1}\right\}} \frac{\mathrm{d}_{G^{\prime}}(x)+\mathrm{d}_{G^{\prime}}(y)}{r_{G^{\prime}}(x, y)}=\sum_{i=2}^{q} \frac{\mathrm{d}_{G^{\prime}}\left(v_{1}\right)+\mathrm{d}_{G^{\prime}}\left(u_{i}\right)}{r_{G^{\prime}}\left(v_{1}, u_{i}\right)}+\sum_{i=2}^{q} \frac{\mathrm{d}_{G^{\prime}}\left(v_{2}\right)+\mathrm{d}_{G^{\prime}}\left(u_{i}\right)}{r_{G^{\prime}}\left(v_{2}, u_{i}\right)}+\cdots+\sum_{i=2}^{q} \frac{\mathrm{d}_{G^{\prime}}\left(v_{p}\right)+\mathrm{d}_{G^{\prime}}\left(u_{i}\right)}{r_{G^{\prime}}\left(v_{p}, u_{i}\right)}$,

and then, we have

$$
\begin{aligned}
& \sum_{i=2}^{q} \frac{\mathrm{d}_{G^{\prime}}\left(v_{1}\right)+\mathrm{d}_{G^{\prime}}\left(u_{i}\right)}{r_{G^{\prime}}\left(v_{1}, u_{i}\right)}=\frac{s_{1}+\cdots+s_{p}+t_{2}+6}{r_{q}^{1}}+\frac{s_{1}+\cdots+s_{p}+t_{3}+6}{r_{q}^{2}}+\cdots+\frac{s_{1}+\cdots+s_{p}+t_{q}+6}{r_{q}^{q-1}}, \\
& \sum_{i=2}^{q} \frac{\mathrm{d}_{G^{\prime}}\left(v_{2}\right)+\mathrm{d}_{G^{\prime}}\left(u_{i}\right)}{r_{G^{\prime}}\left(v_{2}, u_{i}\right)}=\frac{t_{2}+4}{r_{p}^{1}+r_{q}^{1}}+\frac{t_{3}+4}{r_{p}^{1}+r_{q}^{2}}+\cdots+\frac{t_{q}+4}{r_{p}^{1}+r_{q}^{q-1}}, \\
& \ldots \\
& \sum_{i=2}^{q} \frac{\mathrm{d}_{G^{\prime}}\left(v_{p}\right)+\mathrm{d}_{G^{\prime}}\left(u_{i}\right)}{r_{G^{\prime}}\left(v_{p}, u_{i}\right)}=\frac{t_{2}+4}{r_{p}^{p-1}+r_{q}^{1}}+\frac{t_{3}+4}{r_{p}^{p-1}+r_{q}^{2}}+\cdots+\frac{t_{q}+4}{r_{p}^{p-1}+r_{q}^{q-1}} .
\end{aligned}
$$


Thus,

$$
\sum_{x \in V\left(C_{p}\right)} \frac{\mathrm{d}_{G^{\prime}}(x)+\mathrm{d}_{G^{\prime}}(y)}{r_{G^{\prime}}(x, y)}=-\sum_{x \in V\left(C_{q}\right) \backslash\left\{v_{1}\right\}} \sum_{y \in V\left(C_{q}\right) \backslash\left\{v_{1}\right\}} \frac{\mathrm{d}_{G}(x)+\mathrm{d}_{G}(y)}{r_{G}(x, y)}=\sum_{i=2}^{p} \sum_{j=1}^{q-1} \frac{s_{i}}{r_{q}^{j}}-\sum_{i=2}^{p} \sum_{j=1}^{q-1} \frac{s_{i}}{r_{p}^{i-1}+r_{q}^{j}}>0 .
$$

(7) For $x \in V\left(C_{p}\right), y \in V\left(G_{p}^{j}\right) \backslash\left\{u_{j}\right\}$ of $G$ and $G^{\prime}$, respectively, simulating the abovementioned calculation method of (6), we get the similar conclusion,

$$
\sum_{j=2}^{q} \sum_{x \in V\left(C_{p}\right)} \frac{\mathrm{d}_{G^{\prime}}(x)+\mathrm{d}_{G^{\prime}}(y)}{r_{G^{\prime}}(x, y)}-\sum_{j=2}^{q} \sum_{x \in V\left(G_{p}^{j}\right) \backslash\left\{u_{j}\right\}} \frac{\mathrm{d}_{G}(x)+\mathrm{d}_{G}(y)}{r_{G}(x, y)}=\sum_{i=2}^{p} \sum_{j=2}^{q} \frac{s_{i} \cdot t_{j}^{j}}{r_{q}^{j-1}+1}-\sum_{i=2}^{p} \sum_{j=2}^{q} \frac{s_{i} \cdot t_{j}}{r_{p}^{i-1}+r_{q}^{j-1}+1}>0 .
$$

(8) Obviously,

$$
\begin{aligned}
& \sum_{x, y \in V\left(C_{q}\right) \backslash\left\{v_{1}\right\}} \frac{\mathrm{d}_{G}(x)+\mathrm{d}_{G}(y)}{r_{G}(x, y)}+\sum_{j=2}^{q} \sum_{x \in V\left(C_{q}\right) \backslash\left\{v_{1}\right\} y \in V\left(G_{p}^{j}\right) \backslash\left\{u_{j}\right\}} \frac{\mathrm{d}_{G}(x)+\mathrm{d}_{G}(y)}{r_{G}(x, y)} \\
& +\sum_{j=2}^{q} \sum_{x, y \in V\left(G_{p}^{j}\right) \backslash\left\{u_{j}\right\}} \frac{\mathrm{d}_{G}(x)+\mathrm{d}_{G}(y)}{r_{G}(x, y)}+\sum_{i, j=2 i \neq j}^{q} \sum_{x \in V\left(G_{q}^{i}\right) \backslash\left\{u_{i}\right\} y \in V\left(G_{q}^{j}\right) \backslash\left\{u_{j}\right\}} \frac{\mathrm{d}_{G}(x)+\mathrm{d}_{G}(y)}{r_{G}(x, y)}, \\
& =\sum_{x, y \in V\left(C_{q}\right) \backslash\left\{v_{1}\right\}} \frac{\mathrm{d}_{G^{\prime}}(x)+\mathrm{d}_{G^{\prime}}(y)}{r_{G^{\prime}}(x, y)}+\sum_{j=2}^{q} \sum_{x \in V\left(C_{q}\right) \backslash\left\{v_{1}\right\} y \in V\left(G_{p}^{j}\right) \backslash\left\{u_{j}\right\}} \frac{\mathrm{d}_{G^{\prime}}(x)+\mathrm{d}_{G^{\prime}}(y)}{r_{G^{\prime}}(x, y)} \\
& +\sum_{j=2}^{q} \sum_{x, y \in V\left(G_{p}^{j}\right) \backslash\left\{u_{j}\right\}} \frac{\mathrm{d}_{G^{\prime}}(x)+\mathrm{d}_{G^{\prime}}(y)}{r_{G^{\prime}}(x, y)}+\sum_{i, j=2 i \neq j}^{q} \sum_{x \in V\left(G_{q}^{i}\right) \backslash\left\{u_{i}\right\} y \in V\left(G_{q}^{j}\right) \backslash\left\{u_{j}\right\}} \frac{\mathrm{d}_{G^{\prime}}(x)+\mathrm{d}_{G^{\prime}}(y)}{r_{G^{\prime}}(x, y)} .
\end{aligned}
$$

Thus, combing (1)-(8), we get $\operatorname{RDR}\left(G^{\prime}\right)-\operatorname{RDR}(G)>0$, and the results are as follows.

Corollary 1. If $G^{\prime}$ can be obtained from $G$ by a cycle-lifting transformation, see Figure 3 , and $\sum_{j=2}^{q} t_{j}>0$, then $R D R(G)<R D R\left(G^{\prime}\right)$.

2.3. Cycle-Shrinking Transformation. Let $G=S_{n}^{p, q}$, which is obtained by coalescing $C_{p}$ and $C_{q}$ at the vertex $v_{1}$ and attaching $n-p-q+1$ pendent edges to the vertex $v_{1}$. We note that $p, q \geq 3, p+q \leq n+1$. Deleting the edges $v_{2} v_{3}, \ldots, v_{p-1} v_{p}$ and adding the edges $v_{3} v_{1}, v_{4} v_{1}, \ldots$, $v_{p-1} v_{1}, v_{2} v_{p}$, we get $G^{\prime}=S_{n}^{3, q}$ This operation is called cycleshrinking transformation. It is denoted by $(w(w \prime)$, resp), the set of pendent vertices of $\left(G\left(G^{\prime}\right)\right.$, resp). Let $|w|=k \geq 0 ; w^{\prime}$ can be partitioned into two subsets: one has $k$ vertices, which is naturally corresponding to $w$ (also denoted by $w$ ), and another has $p-3$ vertices, denoted by $\widetilde{w}$.

Lemma 4. Let $G=S_{n}^{p, q}(p \geq 4, p+q \leq n+1)$; if $G^{\prime}$ can be obtained from $G$ by a cycle-shrinking transformation, see Figure 4, then $\operatorname{RDR}(G)<R D R\left(G^{\prime}\right)$.

Proof. Let $G$ and $G^{\prime}$ be as shown in Figure 4. By the definition of $\operatorname{RDR}(G)$, 


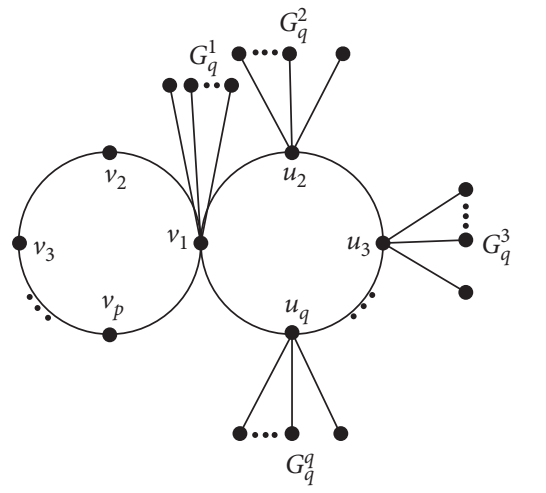

G

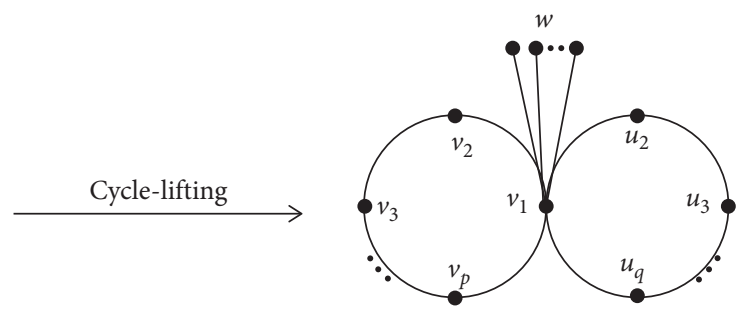

$G^{\prime}$

FIgURE 3: The cycle-lifting transformation.
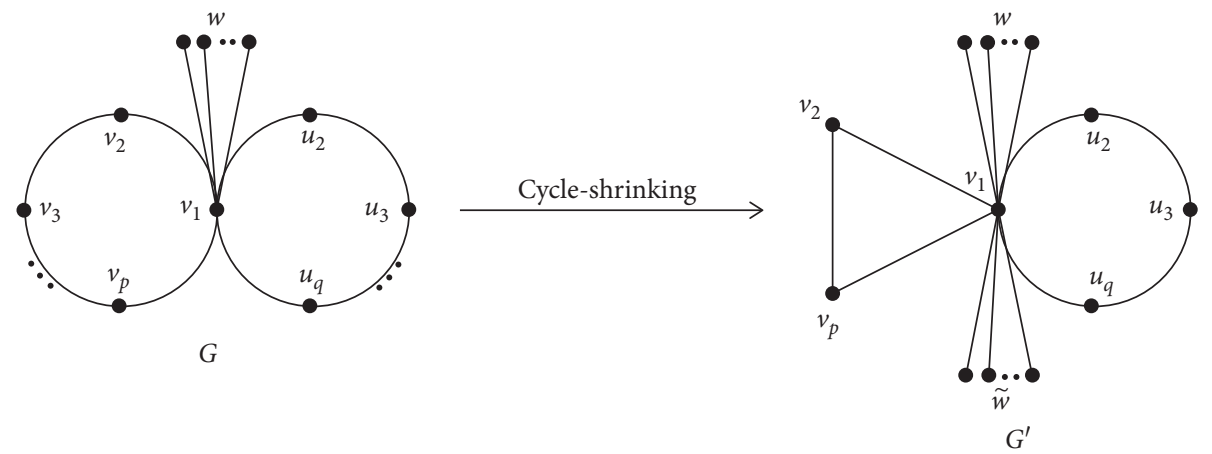

FIgURE 4: The cycle-shrinking transformation.

$$
\begin{aligned}
& \operatorname{RDR}(G)=\sum_{x, y \in w} \frac{\mathrm{d}_{G}(x)+\mathrm{d}_{G}(y)}{r_{G}(x, y)}+\sum_{x \in w} \frac{\mathrm{d}_{G}\left(v_{1}\right)+\mathrm{d}_{G}(x)}{r_{G}\left(v_{1}, y\right)}+\sum_{x \in w y \in\left\{v_{2}, v_{p}\right\}} \frac{\mathrm{d}_{G}(x)+\mathrm{d}_{G}(y)}{r_{G}(x, y)}+\sum_{x \in w \in V\left(C_{p}\right) \backslash\left\{v_{1}, v_{2}, v_{p}\right\}} \frac{\mathrm{d}_{G}(x)+\mathrm{d}_{G}(y)}{r_{G}(x, y)} \\
& +\sum_{x \in w y \in V\left(C_{q}\right) \backslash\left\{v_{1}\right\}} \frac{\mathrm{d}_{G}(x)+\mathrm{d}_{G}(y)}{r_{G}(x, y)}+\sum_{x \in\left\{v_{2}, v_{p}\right\}} \frac{\mathrm{d}_{G}\left(v_{1}\right)+\mathrm{d}_{G}(x)}{r_{G}\left(v_{1}, y\right)}+\sum_{x \in V\left(C_{p}\right) \backslash\left\{v_{1}, v_{2}, v_{p}\right\}} \frac{\mathrm{d}_{G}\left(v_{1}\right)+\mathrm{d}_{G}(x)}{r_{G}\left(v_{1}, y\right)} \\
& +\sum_{x \in V\left(C_{q}\right) \backslash\left\{v_{1}\right\}} \frac{\mathrm{d}_{G}\left(v_{1}\right)+\mathrm{d}_{G}(x)}{r_{G}\left(v_{1}, x\right)}+\frac{\mathrm{d}_{G}\left(v_{2}\right)+\mathrm{d}_{G}\left(v_{p}\right)}{r_{G}\left(v_{2}, v_{p}\right)}+\sum_{x \in\left\{v_{2}, v_{p}\right\}} \sum_{y \in V\left(C_{p}\right) \backslash\left\{v_{1}, v_{2}, v_{p}\right\}} \frac{\mathrm{d}_{G}(x)+\mathrm{d}_{G}(y)}{r_{G}(x, y)} \\
& +\sum_{x \in\left\{v_{2}, v_{p}\right\}} \frac{\mathrm{d}_{G}(x)+\mathrm{d}_{G}(y)}{r_{G}(x, y)} \\
& \left.+\sum_{x, y \in V\left(C_{p}\right) \backslash\left\{v_{1}, v_{2}, v_{p}\right\}} \frac{\mathrm{d}_{G}(x)+\mathrm{d}_{G}(y)}{r_{G}(x, y)}+\sum_{x \in V\left(C_{p}\right) \backslash\left\{v_{1}, v_{2}, v_{p}\right\}}\right\}_{y \in V\left(C_{q}\right) \backslash\left\{v_{1}\right\}} \frac{\mathrm{d}_{G}(x)+\mathrm{d}_{G}(y)}{r_{G}(x, y)}+\sum_{x, y \in V\left(C_{q}\right) \backslash\left\{v_{1}\right\}} \frac{\mathrm{d}_{G}(x)+\mathrm{d}_{G}(y)}{r_{G}(x, y)},
\end{aligned}
$$




$$
\begin{aligned}
\operatorname{RDR}\left(G^{\prime}\right)= & \sum_{x, y \in w} \frac{\mathrm{d}_{G^{\prime}}(x)+\mathrm{d}_{G^{\prime}}(y)}{r_{G^{\prime}}(x, y)}+\sum_{x \in w} \frac{\mathrm{d}_{G^{\prime}}\left(v_{1}\right)+\mathrm{d}_{G^{\prime}}(x)}{r_{G^{\prime}}\left(v_{1}, x\right)}+\sum_{x \in w y \in\left\{v_{2}, v_{p}\right\}} \frac{\mathrm{d}_{G^{\prime}}(x)+\mathrm{d}_{G^{\prime}}(y)}{r_{G^{\prime}}(x, y)}+\sum_{x \in w \in \tilde{w}} \frac{\mathrm{d}_{G^{\prime}}(x)+\mathrm{d}_{G^{\prime}}(y)}{r_{G^{\prime}}(x, y)} \\
& +\sum_{x \in w y \in V\left(C_{q}\right) \backslash\left\{v_{1}\right\}} \frac{\mathrm{d}_{G^{\prime}}(x)+\mathrm{d}_{G^{\prime}}(y)}{r_{G^{\prime}}(x, y)}+\sum_{x \in\left\{v_{2}, v_{p}\right\}} \frac{\mathrm{d}_{G^{\prime}}\left(v_{1}\right)+\mathrm{d}_{G^{\prime}}(x)}{r_{G^{\prime}}\left(v_{1}, x\right)}+\sum_{x \in \widetilde{w}} \frac{\mathrm{d}_{G^{\prime}}\left(v_{1}\right)+\mathrm{d}_{G^{\prime}}(x)}{r_{G^{\prime}}\left(v_{1}, x\right)} \\
& +\sum_{x \in V\left(C_{q}\right) \backslash\left\{v_{1}\right\}} \frac{\mathrm{d}_{G^{\prime}}\left(v_{1}\right)+\mathrm{d}_{G^{\prime}}(x)}{r_{G^{\prime}}\left(v_{1}, x\right)}+\frac{\mathrm{d}_{G^{\prime}}\left(v_{2}\right)+\mathrm{d}_{G^{\prime}}\left(v_{p}\right)}{r_{G^{\prime}}\left(v_{2}, v_{p}\right)} \\
& \left.+\sum_{x \in\left\{v_{2}, v_{p}\right\}} \frac{\mathrm{d}_{G^{\prime}}(x)+\mathrm{d}_{G^{\prime}}(y)}{r_{G^{\prime}}(x, y)}+\sum_{x \in\left\{v_{2}, v_{p}\right\}}\right\} y \in V\left(C_{q}\right) \backslash\left\{v_{1}\right\} \\
& +\sum_{x \in \tilde{w} y \in V\left(C_{q}\right) \backslash\left\{v_{1}\right\}} \frac{\mathrm{d}_{G^{\prime}}(x)+\mathrm{d}_{G^{\prime}}(y)}{r_{G^{\prime}}(x, y)}+\sum_{x, y \in V\left(C_{q}\right) \backslash\left\{v_{1}\right\}} \frac{\mathrm{d}_{G^{\prime}}(x)+\mathrm{d}_{G^{\prime}}(y)}{r_{G^{\prime}}(x, y)} .
\end{aligned}
$$

(1) Noting that $\mathrm{d}_{G}(x)+\mathrm{d}_{G}(y)=\mathrm{d}_{G^{\prime}}(x)+\mathrm{d}_{G^{\prime}}(y)$, $r_{G}(x, y)=r_{G^{\prime}}(x, y)$ for any $x, y \in w$ of $G$ and $G^{\prime}$, respectively, we have

$$
\sum_{x, y \in w} \frac{\mathrm{d}_{G}(x)+\mathrm{d}_{G}(y)}{r_{G}(x, y)}=\sum_{x, y \in w} \frac{\mathrm{d}_{G^{\prime}}(x)+\mathrm{d}_{G^{\prime}}(y)}{r_{G^{\prime}}(x, y)} .
$$

(2) Noting that $\mathrm{d}_{G}\left(v_{1}\right)+\mathrm{d}_{G}(x)=k+5, \quad \mathrm{~d}_{G^{\prime}}\left(v_{1}\right)+$ $\mathrm{d}_{G^{\prime}}(x)=k+p+2, r_{G}\left(v_{1}, x\right)=r_{G^{\prime}}\left(v_{1}, x\right)=1$ for $x \in w$ in $G$ and $G^{\prime}$, respectively, we have

$$
\begin{gathered}
\sum_{x \in w} \frac{\mathrm{d}_{G^{\prime}}\left(v_{1}\right)+\mathrm{d}_{G^{\prime}}(x)}{r_{G^{\prime}}\left(v_{1}, x\right)}-\sum_{x \in w} \frac{\mathrm{d}_{G}\left(v_{1}\right)+\mathrm{d}_{G}(x)}{r_{G}\left(v_{1}, x\right)} \\
=k(k+p+2)-k(k+5)=k(p-3) .
\end{gathered}
$$

(3) Noting that $\mathrm{d}_{G}(x)+\mathrm{d}_{G}(y)=\mathrm{d}_{G^{\prime}}(x)+\mathrm{d}_{G^{\prime}}(y)=3$, $r_{G}(x, y)=((p-1) / p)+1 \geq r_{G^{\prime}}(x, y)=(2 / 3)+1$ for any $x \in w, y \in\left\{v_{2}, v_{p}\right\}$ in $G$ and $x \in w$, $y \in\left\{v_{2}, v_{p}\right\}$ in $G^{\prime}$, we have

$$
\sum_{x \in w y \in\left\{v_{2}, v_{p}\right\}} \frac{\mathrm{d}_{G^{\prime}}(x)+\mathrm{d}_{G^{\prime}}(y)}{r_{G^{\prime}}(x, y)}-\sum_{x \in w y \in\left\{v_{2}, v_{p}\right\}} \frac{\mathrm{d}_{G}(x)+\mathrm{d}_{G}(y)}{r_{G}(x, y)}>0 .
$$

(4) Noting that $\mathrm{d}_{G}(x)+\mathrm{d}_{G}(y)=3, r_{G}(x, y)=$ $r_{G}\left(v_{i}, v_{1}\right)+1>2(3 \leq i \leq p-1) \quad$ for $\quad x \in w, y \in$ $V\left(C_{p}\right) \backslash\left\{v_{1}, v_{2}, v_{p}\right\} \quad$ and $\quad \mathrm{d}_{G^{\prime}}(x)+\mathrm{d}_{G^{\prime}}(y)=2$, $r_{G^{\prime}}(x, y)=2$ for $x \in w, y \in \widetilde{w}$, we have

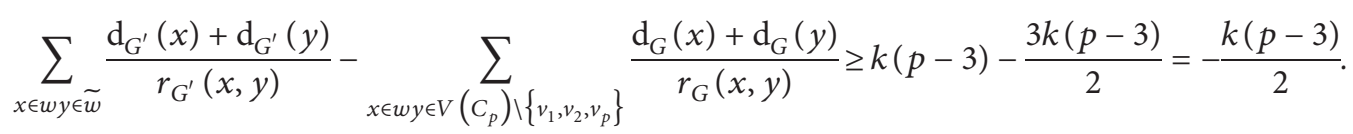

Thus, combing (2) and (4), we have

$$
\begin{aligned}
& \left(\sum_{x \in w} \frac{\mathrm{d}_{G^{\prime}}\left(v_{1}\right)+\mathrm{d}_{G^{\prime}}(x)}{r_{G^{\prime}}\left(v_{1}, x\right)}-\sum_{x \in w} \frac{\mathrm{d}_{G}\left(v_{1}\right)+\mathrm{d}_{G}(x)}{r_{G}\left(v_{1}, x\right)}\right)+\left(\sum_{x \in w y \in \widetilde{w}} \frac{\mathrm{d}_{G^{\prime}}(x)+\mathrm{d}_{G^{\prime}}(y)}{r_{G^{\prime}}(x, y)}-\sum_{x \in w y \in V\left(C_{p}\right) \backslash\left\{v_{1}, v_{2}, v_{p}\right\}} \frac{\mathrm{d}_{G}(x)+\mathrm{d}_{G}(y)}{r_{G}(x, y)}\right) \\
& \quad \geq \frac{k(p-3)}{2} \geq 0 .
\end{aligned}
$$


(5) Noting that $\mathrm{d}_{G}(x)+\mathrm{d}_{G}(y)=\mathrm{d}_{G^{\prime}}(x)+\mathrm{d}_{G^{\prime}}(y)$, $r_{G}(x, y)=r_{G^{\prime}}(x, y)$ for any $x \in w, y \in V\left(C_{q}\right) \backslash\left\{v_{1}\right\}$ of $G$ and $G^{\prime}$, respectively, we have

$\sum_{x \in w y \in V\left(C_{q}\right) \backslash\left\{v_{1}\right\}} \frac{\mathrm{d}_{G}(x)+\mathrm{d}_{G}(y)}{r_{G}(x, y)}=\sum_{x \in w y \in V\left(C_{q}\right) \backslash\left\{v_{1}\right\}} \frac{\mathrm{d}_{G^{\prime}}(x)+\mathrm{d}_{G^{\prime}}(y)}{r_{G^{\prime}}(x, y)}$.

(6) Noting that $\mathrm{d}_{G}\left(v_{1}\right)+\mathrm{d}_{G}(x)=k+6, \quad r_{G}\left(v_{1}, x\right)=$ $((p-1) / p) \quad$ and $\quad \mathrm{d}_{G^{\prime}}\left(v_{1}\right)+\mathrm{d}_{G^{\prime}}(x)=k+p+$ 3, $r_{G^{\prime}}\left(v_{1}, x\right)=(2 / 3)$ for $x \in\left\{v_{2}, v_{p}\right\}$ of $G$ and $G^{\prime}$, respectively, we have

$$
\begin{aligned}
& \sum_{x \in\left\{v_{2}, v_{p}\right\}} \frac{\mathrm{d}_{G^{\prime}}\left(v_{1}\right)+\mathrm{d}_{G^{\prime}}(x)}{r_{G^{\prime}}\left(v_{1}, x\right)}-\sum_{x \in\left\{v_{2}, v_{p}\right\}} \frac{\mathrm{d}_{G}\left(v_{1}\right)+\mathrm{d}_{G}(x)}{r_{G}\left(v_{1}, x\right)} \\
& =3(k+p+3)-\frac{2 p(k+6)}{p-1}=\frac{(k+3 p+3)(p-3)}{p-1} .
\end{aligned}
$$

(7) Noting that $\mathrm{d}_{G}\left(v_{1}\right)+\mathrm{d}_{G}(x)=k+6$, $r_{G}\left(v_{1}, x\right)=r_{G}\left(v_{1}, v_{i}\right)>1(3 \leq i \leq p-1)$ for $x \in V\left(C_{p}\right) \backslash$ $\left\{v_{1}, v_{2}, v_{p}\right\}$ of $G$ and $\mathrm{d}_{G^{\prime}}\left(v_{1}\right)+\mathrm{d}_{G^{\prime}}(x)=k+p+2$, $r_{G^{\prime}}\left(v_{1}, x\right)=1$ for $x \in \tilde{w}$ of $G^{\prime}$, we have

$$
\begin{gathered}
\sum_{x \in \widetilde{w}} \frac{\mathrm{d}_{G^{\prime}}\left(v_{1}\right)+\mathrm{d}_{G^{\prime}}(x)}{r_{G^{\prime}}\left(v_{1}, x\right)}-\sum_{x \in V\left(C_{p}\right) \backslash\left\{v_{1}, v_{2}, v_{p}\right\}} \frac{\mathrm{d}_{G}\left(v_{1}\right)+\mathrm{d}_{G}(x)}{r_{G}\left(v_{1}, x\right)} \\
\geq(p-3)(k+p+2)-(p-3)(k+6)=(p-3)(p-4) .
\end{gathered}
$$

(8) Noting that $\mathrm{d}_{G}\left(v_{1}\right)+\mathrm{d}_{G}(x)=k+6, \mathrm{~d}_{G^{\prime}}\left(v_{1}\right)+$ $\mathrm{d}_{G^{\prime}}(x)=k+p+3, r_{G}\left(v_{1}, x\right)=r_{G^{\prime}}\left(v_{1}, x\right) \quad$ for $x \in V\left(C_{q}\right) \backslash\left\{v_{1}\right\}$ of $G$ and $G^{\prime}$, respectively, we have

$$
\begin{aligned}
& \sum_{x \in V\left(C_{q}\right) \backslash\left\{v_{1}\right\}} \frac{\mathrm{d}_{G^{\prime}}\left(v_{1}\right)+\mathrm{d}_{G^{\prime}}(x)}{r_{G^{\prime}}\left(v_{1}, x\right)}-\sum_{x \in V\left(C_{q}\right) \backslash\left\{v_{1}\right\}} \frac{\mathrm{d}_{G}\left(v_{1}\right)+\mathrm{d}_{G}(x)}{r_{G}\left(v_{1}, x\right)} \\
& =(p-3) \sum_{j=2}^{q} \frac{1}{r_{G}\left(v_{1}, u_{j}\right)} .
\end{aligned}
$$

(9) Noting that $\mathrm{d}_{G}\left(v_{2}\right)+\mathrm{d}_{G}\left(v_{p}\right)=4, \quad r_{G}\left(v_{2}, v_{p}\right)=$ $((2(p-2)) / p) \quad$ and $\quad \mathrm{d}_{G^{\prime}}\left(v_{2}\right)+\mathrm{d}_{G^{\prime}}\left(v_{p}\right)=4$, $r_{G^{\prime}}\left(v_{2}, v_{p}\right)=(2 / 3)$, we have

$$
\frac{\mathrm{d}_{G^{\prime}}\left(v_{2}\right)+\mathrm{d}_{G^{\prime}}\left(v_{p}\right)}{r_{G^{\prime}}\left(v_{2}, v_{p}\right)}-\frac{\mathrm{d}_{G}\left(v_{2}\right)+\mathrm{d}_{G}\left(v_{p}\right)}{r_{G}\left(v_{2}, v_{p}\right)}=6-\frac{2 p}{p-2}=\frac{4 p-12}{p-2}>0 .
$$

(10) Noting that $\mathrm{d}_{G}(x)+\mathrm{d}_{G}(y)=4$ for $x \in\left\{v_{2}, v_{p}\right\}$, $y \in V\left(C_{p}\right) \backslash\left\{v_{1}, v_{2}, v_{p}\right\} \quad$ of $\quad G \quad$ and $\quad \mathrm{d}_{G^{\prime}}(x)+$ $\mathrm{d}_{G^{\prime}}(y)=3, \quad r_{G^{\prime}}(x, y)=r_{G^{\prime}}\left(v_{2}, v_{1}\right)+1=$ $(2 / 3)+1=(5 / 3)$ for $x \in\left\{v_{2}, v_{p}\right\}, y \in \widetilde{w}$ of $G^{\prime}$, by the symmetry between $v_{2}$ and $v_{p}$, we have

$$
\begin{aligned}
\sum_{x \in\left\{v_{2}, v_{p}\right\}} \frac{\mathrm{d}_{G^{\prime}}(x)+\mathrm{d}_{G^{\prime}}(y)}{r_{G^{\prime}}(x, y)}-\sum_{x \in\left\{v_{2}, v_{p}\right\}} \sum_{y \in V\left(C_{p}\right) \backslash\left\{v_{1}, v_{2}, v_{p}\right\}} \frac{\mathrm{d}_{G}(x)+\mathrm{d}_{G}(y)}{r_{G}(x, y)} & =2 \sum_{i=3}^{p-1} \frac{3}{r_{G^{\prime}}\left(v_{2}, v_{1}\right)+1}-2 \sum_{i=3}^{p-1} \frac{4}{r_{G}\left(v_{2}, v_{i}\right)}, \\
& =\frac{18(p-3)}{5}-\left(\frac{8 p}{p-1}+2 \sum_{i=4}^{p-1} \frac{4}{r_{G}\left(v_{2}, v_{i}\right)}\right) .
\end{aligned}
$$

Since $r_{G}\left(v_{2}, v_{i}\right) \geq r_{G} \quad\left(v_{2}, v_{4}\right)=((2(p-2)) / p)$ Thus, $(4 \leq i \leq p-1)$, if $p \geq 12$, then $((2(p-2)) / p) \geq$ $(5 / 3)$, and

$$
2 \sum_{i=4}^{p-1} \frac{4}{r_{G}\left(v_{2}, v_{i}\right)} \leq \frac{24(p-4)}{5} .
$$

$$
\sum_{x \in\left\{v_{2}, v_{p}\right\}} \frac{\mathrm{d}_{G^{\prime}}(x)+\mathrm{d}_{G^{\prime}}(y)}{r_{G^{\prime}}(x, y)}-\sum_{x \in\left\{v_{2}, v_{p}\right\}} \sum_{y \in V\left(C_{p}\right) \backslash\left\{v_{1}, v_{2}, v_{p}\right\}} \frac{\mathrm{d}_{G}(x)+\mathrm{d}_{G}(y)}{r_{G}(x, y)} \geq \frac{18(p-3)}{5}-\left(\frac{8 p}{p-1}+\frac{24(p-4)}{5}\right) .
$$


(11) Noting that $\mathrm{d}_{G}(x)+\mathrm{d}_{G}(y)=4$ for $x \in\left\{v_{2}, v_{p}\right\}$, $y \in V\left(C_{q}\right) \backslash\left\{v_{1}\right\}$ of $G, \mathrm{~d}_{G^{\prime}}(x)+\mathrm{d}_{G^{\prime}}(y)=4$ for $x \in\left\{v_{2}, v_{p}\right\}, y \in V\left(C_{q}\right) \backslash\left\{v_{1}\right\}$ of $G^{\prime}$, and $r_{G}(x, y)=$ $r_{G}\left(v_{2}, v_{1}\right)+r_{G}\left(v_{1}, u_{i}\right)=((p-1) / p)+r_{G}\left(v_{1}, u_{i}\right) \geq$
$r_{G^{\prime}}(x, y)=r_{G^{\prime}}\left(v_{2}, v_{1}\right)+r_{G^{\prime}}\left(v_{1}, u_{i}\right)=(2 / 3)+r_{G^{\prime}}\left(v_{1}\right.$, $\left.u_{i}\right)(2 \leq i \leq q)$, by symmetry between $v_{2}$ and $v_{p}$, we have

$$
\sum_{x \in\left\{v_{2}, v_{p}\right\}} \frac{\mathrm{d}_{G^{\prime}}(x)+\mathrm{d}_{G^{\prime}}(y)}{r_{G^{\prime}}(x, y)}-\sum_{x \in\left\{v_{2}, v_{p}\right\}} \sum_{y \in V}\left(C_{q}\right) \backslash\left\{v_{q}\right\} \quad \frac{\mathrm{d}_{G}(x)+\mathrm{d}_{G}(y)}{r_{G}(x, y)} \geq 0 .
$$

(12) Noting that $\mathrm{d}_{G}(x)+\mathrm{d}_{G}(y)=4, r_{G}(x, y)=r_{G}\left(v_{i}\right.$, $\left.v_{j}\right)(3 \leq i<j \leq p-1)$ for $x, y \in V\left(C_{p}\right) \backslash\left\{v_{1}, v_{2}, v_{p}\right\}$ of

$G$ and $\mathrm{d}_{G^{\prime}}(x)+\mathrm{d}_{G^{\prime}}(y)=2, \quad r_{G^{\prime}}(x, y)=2$ for $x, y \in \widetilde{w}$ of $G^{\prime}$, we have

$$
\begin{aligned}
& \sum_{x, y \in \widetilde{w}} \frac{\mathrm{d}_{G^{\prime}}(x)+\mathrm{d}_{G^{\prime}}(y)}{r_{G^{\prime}}(x, y)}-\sum_{x, y \in V\left(C_{p}\right) \backslash\left\{v_{1}, v_{2}, v_{p}\right\}} \frac{\mathrm{d}_{G}(x)+\mathrm{d}_{G}(y)}{r_{G}(x, y)}=\frac{(p-3)(p-4)}{2}-\sum_{i, j=3 i<j}^{p-1} \frac{4}{r_{G}\left(v_{i}, v_{j}\right)}, \\
& \sum_{i, j=3 i<j}^{p-1} \frac{4}{r_{G}\left(v_{i}, v_{j}\right)}=\sum_{i=4}^{p} \frac{\mathrm{d}_{G}\left(v_{3}\right)+\mathrm{d}_{G}\left(v_{i}\right)}{r_{G}\left(v_{3}, v_{i}\right)}+\sum_{i=5}^{p} \frac{\mathrm{d}_{G}\left(v_{4}\right)+\mathrm{d}_{G}\left(v_{i}\right)}{r_{G}\left(v_{4}, v_{i}\right)}+\cdots+\frac{\mathrm{d}_{G}\left(v_{p-2}\right)+\mathrm{d}_{G}\left(v_{p-1}\right)}{r_{G}\left(v_{p-2}, v_{p-1}\right)} .
\end{aligned}
$$

When $j-i=t(i<j)$, we denote $r_{G}\left(v_{i}, v_{j}\right)=r_{t}$, and then, $r_{t}=r_{p-t}=((t(p-t)) / p)$; thus,

$$
\begin{gathered}
\sum_{i=4}^{p} \frac{\mathrm{d}_{G}\left(v_{3}\right)+\mathrm{d}_{G}\left(v_{i}\right)}{r_{G}\left(v_{3}, v_{i}\right)}=\frac{4}{r_{1}}+\frac{4}{r_{2}}+\cdots+\frac{4}{r_{p-4}}, \\
\sum_{i=5}^{p} \frac{\mathrm{d}_{G}\left(v_{4}\right)+\mathrm{d}_{G}\left(v_{i}\right)}{r_{G}\left(v_{4}, v_{i}\right)}=\frac{4}{r_{1}}+\cdots+\frac{4}{r_{p-5}}, \\
\cdots \\
\frac{\mathrm{d}_{G}\left(v_{p-2}\right)+\mathrm{d}_{G}\left(v_{p-1}\right)}{r_{G}\left(v_{p-2}, v_{p-1}\right)}=\frac{4}{r_{1}} .
\end{gathered}
$$

$$
\sum_{i, j=3 i<j}^{p-1} \frac{4}{r_{G}\left(v_{i}, v_{j}\right)}=\frac{4(p-4)}{r_{1}}+\frac{4(p-5)}{r_{2}}+\cdots+\frac{4}{r_{p-4}} \text {. }
$$

Since

$$
r_{1}=((p-1) / p),
$$

$r_{t} \geq r_{2}=((2(p-2)) / p)(2 \leq t \leq p-4)$. If $p \geq 12$, then $((2(p-2)) / p) \geq(5 / 3)$, and

$$
\sum_{i, j=3 i<j}^{p-1} \frac{4}{r_{G}\left(v_{i}, v_{j}\right)} \leq \frac{4 p(p-4)}{p-1}+\frac{6(p-4)(p-5)}{5} .
$$

Thus,

$$
\sum_{x, y \in \widetilde{w}} \frac{\mathrm{d}_{G^{\prime}}(x)+\mathrm{d}_{G^{\prime}}(y)}{r_{G^{\prime}}(x, y)}-\sum_{x, y \in V\left(C_{p}\right) \backslash\left\{v_{1}, v_{2}, v_{p}\right\}} \frac{\mathrm{d}_{G}(x)+\mathrm{d}_{G}(y)}{r_{G}(x, y)} \geq \frac{(p-3)(p-4)}{2}-\left(\frac{4 p(p-4)}{p-1}+\frac{6(p-4)(p-5)}{5}\right) .
$$

When $p \geq 12$, combing (6), (7), (10), and (12), we have 


$$
\begin{aligned}
& \left(\sum_{x \in\left\{v_{2}, v_{p}\right\}} \frac{\mathrm{d}_{G^{\prime}}\left(v_{1}\right)+\mathrm{d}_{G^{\prime}}(x)}{r_{G^{\prime}}\left(v_{1}, x\right)}-\sum_{x \in\left\{v_{2}, v_{p}\right\}} \frac{\mathrm{d}_{G}\left(v_{1}\right)+\mathrm{d}_{G}(x)}{r_{G}\left(v_{1}, x\right)}\right)+\left(\sum_{x \in \widetilde{w}} \frac{\mathrm{d}_{G^{\prime}}\left(v_{1}\right)+\mathrm{d}_{G^{\prime}}(x)}{r_{G^{\prime}}\left(v_{1}, x\right)}-\sum_{x \in V\left(C_{p}\right) \backslash\left\{v_{1}, v_{2}, v_{p}\right\}} \frac{\mathrm{d}_{G}\left(v_{1}\right)+\mathrm{d}_{G}(x)}{r_{G}\left(v_{1}, x\right)}\right) \\
& +\left(\sum_{x \in\left\{v_{2}, v_{p}\right\}} \frac{\mathrm{d}_{G^{\prime}}(x)+\mathrm{d}_{G^{\prime}}(y)}{r_{G^{\prime}}(x, y)}-\sum_{x \in\left\{v_{2}, v_{p}\right\}} \sum_{y \in V\left(C_{p}\right) \backslash\left\{v_{1}, v_{2}, v_{p}\right\}} \frac{\mathrm{d}_{G}(x)+\mathrm{d}_{G}(y)}{r_{G}(x, y)}\right) \\
& +\left(\sum_{x, y \in \widetilde{w}} \frac{\mathrm{d}_{G^{\prime}}(x)+\mathrm{d}_{G^{\prime}}(y)}{r_{G^{\prime}}(x, y)}-\sum_{x, y \in V\left(C_{p}\right) \backslash\left\{v_{1}, v_{2}, v_{p}\right\}} \frac{\mathrm{d}_{G}(x)+\mathrm{d}_{G}(y)}{r_{G}(x, y)}\right) \\
& \geq \frac{(k+3 p+3)(p-3)}{p-1}+\frac{3(p-3)(p-4)}{2}+\frac{18(p-3)}{5}-\frac{4 p(p-2)}{p-1}-\frac{6(p-1)(p-4)}{5} \\
& =\frac{3 p^{3}-22 p^{2}+53 p-114+k(10 p-30)}{10(p-1)} \text {. }
\end{aligned}
$$

(i) Let $f(p)=3 p^{3}-22 p^{2}+53 p-114, p \in[12,+\infty)$; this function is strictly increasing in this interval; thus, $f(p) \geq f(12)=2538>0$.

Thus,

$$
\frac{3 p^{3}-22 p^{2}+33 p-54+k(10 p-30)}{10(p-1)}>0 .
$$

(13) Noting that $\mathrm{d}_{G}(x)+\mathrm{d}_{G}(y)=4, \quad r_{G}(x, y)=$ $r_{G}\left(v_{i}, v_{1}\right)+r_{G}\left(v_{1}, u_{j}\right) \geq 1+r_{G}\left(v_{1}, u_{j}\right)(3 \leq i \leq p-$ $1,2 \leq j \leq q) \quad$ for $\quad x \in V\left(C_{p}\right) \backslash\left\{v_{1}, v_{2}, v_{p}\right\}, y \in$ $V\left(C_{q}\right) \backslash\left\{v_{1}\right\} \quad$ of $G$ and $\mathrm{d}_{G^{\prime}}(x)+\mathrm{d}_{G^{\prime}}(y)=3$, $r_{G^{\prime}}(x, y)=1+r_{G}\left(v_{1}, u_{j}\right)(2 \leq j \leq q)$ for $x, y \in \widetilde{w}$ of $G^{\prime}$, we have

$$
\begin{aligned}
& \left.\sum_{x \in \tilde{w} y \in V\left(C_{q}\right) \backslash\left\{v_{1}\right\}} \frac{\mathrm{d}_{G^{\prime}}(x)+\mathrm{d}_{G^{\prime}}(y)}{r_{G^{\prime}}(x, y)}-\sum_{x \in V\left(C_{p}\right) \backslash\left\{v_{1}, v_{2}, v_{p}\right\}}\right\}_{y \in V\left(C_{q}\right) \backslash\left\{v_{1}\right\}} \frac{\mathrm{d}_{G}(x)+\mathrm{d}_{G}(y)}{r_{G}(x, y)}, \\
& =(p-3) \sum_{j=2}^{q} \frac{3}{1+r_{G}\left(v_{1}, u_{j}\right)}-\sum_{i=3}^{p-1} \sum_{j=2}^{q} \frac{4}{r_{G}\left(v_{i}, v_{1}\right)+r_{G}\left(v_{1}, u_{j}\right)} \\
& \geq-(p-3) \sum_{j=2}^{q} \frac{1}{1+r_{G}\left(v_{1}, u_{j}\right)} \text {. }
\end{aligned}
$$

Thus, combining (8) and (13), we have

$$
\begin{aligned}
& \left(\sum_{x \in V} \frac{\mathrm{d}_{G}\left(v_{1}\right)+\mathrm{d}_{G}(x)}{r_{G}\left(v_{1}, x\right)}-\sum_{x \in V\left(c_{q}\right) \backslash\left\{v_{1}\right\}} \frac{\mathrm{d}_{G}\left(v_{1}\right)+\mathrm{d}_{G}(x)}{r_{G}\left(v_{1}, x\right)}\right)+\left(\sum_{x \in \tilde{w} y \in V\left(C_{q}\right)\left\{v_{1}\right\}} \frac{\mathrm{d}_{G^{\prime}}(x)+\mathrm{d}_{G^{\prime}}(y)}{r_{G^{\prime}}(x, y)}-\sum_{x \in V\left(C_{p}\right) \backslash\left\{v_{1}, v_{2}, v_{p}\right\}} \sum_{y \in V\left(C_{q}\right) \backslash\left\{v_{1}\right\}} \frac{\mathrm{d}_{G}(x)+\mathrm{d}_{G}(y)}{r_{G}(x, y)}\right), \\
& =(p-3) \sum_{j=2}^{q} \frac{1}{r_{G}\left(v_{1}, u_{j}\right)}+\left(-(p-3) \sum_{j=2}^{q} \frac{1}{1+r_{G}\left(v_{1}, u_{j}\right)}\right)>0 .
\end{aligned}
$$




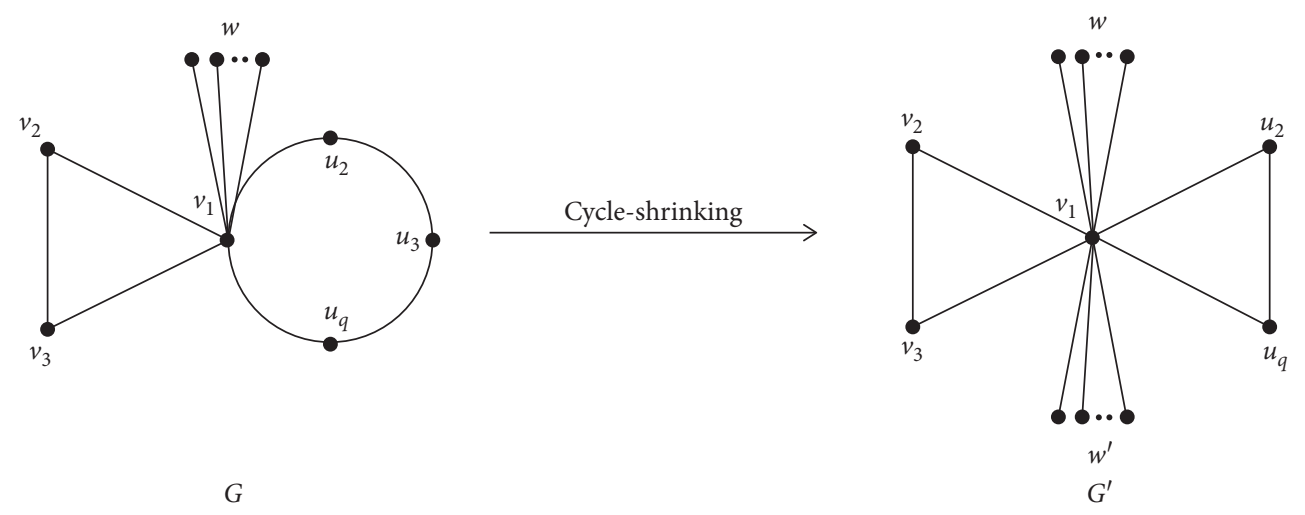

FIGURE 5: The cycle-shrinking transformation.

So, if $p \geq 12$, we get $\operatorname{RDR}\left(G^{\prime}\right)-\operatorname{RDR}(G)>0$, and the results are as follows.

If $4 \leq p \leq 11, \quad B_{n}\left(G_{0}\right)=\left\{S_{n}^{4, q}, S_{n}^{5, q}, S_{n}^{6, q}, S_{n}^{7, q}, S_{n}^{8, q}, \quad S_{n}^{9, q}\right.$, $\left.S_{n}^{10, q}, S_{n}^{11, q}\right\}$. By comparison of direct calculation, we can obtain $\operatorname{RDR}\left(S_{n}^{3, q}\right)>\operatorname{RDR}\left(S_{n}^{p, q}\right)$.

The proof is completed.

Corollary 2. If $G^{\prime}$ can be obtained from $G$ by a cycleshrinking transformation, see Figure 5, and $q \geq 4$, then $R D R(G)<R D R\left(G^{\prime}\right)$.

\section{Maximum Reciprocal Degree Resistance Distance Index of Bicyclic Graphs}

Let $G \in \mathscr{B}_{n}$; applying a sequence of edge-lifting transformations, cycle-lifting transformations, and cycle-shrinking transformations to $G$, we can obtain a new graph $G^{\prime}$, denoted by $S_{n}^{3,3}$, see Figure 5 . The next theorem discusses the maximum reciprocal degree resistance distance index in $\mathscr{B}_{n}$.

Theorem 1. Let $G \in \mathscr{B}_{n}$; then, $R D R(G) \leq R D R\left(S_{n}^{3,3}\right)$, with equality holding if and only if $G \cong S_{n}^{3,3}$.

Proof. Let $G \in \mathscr{B}_{n}$ such that the $\operatorname{RDR}(G)$ is as big as possible. By Lemma 2-4, Corollary 2, we have $\operatorname{RDR}(G) \leq$ $\operatorname{RDR}\left(S_{n}^{3,3}\right)$, and equality holds if and only if $G \cong S_{n}^{3,3}$.

\section{Conclusions}

In this paper, we characterize the maximum reciprocal degree resistance distance index among all bicyclic graphs without common edges of order $n$ by using three types of transformation which keep the reciprocal degree resistance distance index increasing: edge-lifting transformation, cycle-lifting transformation, and cycle-shrinking transformation.

\section{Data Availability}

The data used to support the findings of this study are included within the article.

\section{Conflicts of Interest}

The authors declare that there are no conflicts of interest regarding the publication of this paper.

\section{Acknowledgments}

This work was jointly supported by the National Natural Science Foundation of China (11871077), the Natural Science Foundation of Anhui Province (1808085MA04 and 1908085MC62), and the Natural Science Foundation of Department of Education of Anhui Province (KJ2017A362).

\section{References}

[1] K. Xu, M. Liu, K. C. Das, I. Gutman, and B. Furtula, "A survey on graphs extremal with respect to distancebased topological indices," MATCH Communications in Mathematical and in Computer Chemistry, vol. 71, pp. 461-508, 2014.

[2] I. Gutman, "Degree-based topological indices," Croatica Chemica Acta, vol. 86, no. 4, pp. 351-361, 2013.

[3] H. Wiener, "Structural determination of paraffin boiling points," Journal of the American Chemical Society, vol. 69, no. 1, pp. 17-20, 1947.

[4] B. Furtula, I. Gutman, and V. Katanić, "Three-center Harary index and its applications," Iranian Journal Of Mathematical Chemistry, vol. 7, pp. 61-68, 2016.

[5] D. Bonchev, A. T. Balaban, X. Liu, and D. J. Klein, "Molecular cyclicity and centricity of polycyclic graphs. I. Cyclicity based on resistance distances or reciprocal distances," International Journal of Quantum Chemistry, vol. 50, no. 1, pp. 1-20, 1994.

[6] D. J. Klein and M. Randić, "Resistance distance," Journal of Mathematical Chemistry, vol. 12, no. 1, pp. 81-95, 1993.

[7] I. Gutman, B. Ruščić, N. Trinajstić, and C. F. Wilcox, "Graph theory and molecular orbitals. xii. Acyclic polyenes," The Journal of Chemical Physics, vol. 62, no. 9, pp. 3399-3045, 1975.

[8] I. Gutman and N. Trinajstić, "Graph theory and molecular orbitals. Total $\varphi$-electron energy of alternant hydrocarbons," Chemical Physics Letters, vol. 17, no. 4, pp. 535-538, 1972.

[9] A. A. Dobrynin and A. A. Kochtova, "Degree distance of a graph: a degree analogue of the Wiener index," Journal of Chemical Information and Computer Sciences, vol. 34, 1994.

[10] I. Gutman, "Selected properties of the Schultz molecular topological index," Journal of Chemical Information and Computer Sciences, vol. 34, no. 5, pp. 1087-1089, 1994. 
[11] Y. Alizadeh, A. Iranmanesh, and T. Došlić, "Additively weighted Harary index of some composite graphs," Discrete Mathematics, vol. 313, no. 1, pp. 26-34, 2013.

[12] H. Hua and S. Zhang, "On the reciprocal degree distance of graphs," Discrete Applied Mathematics, vol. 160, no. 7-8, pp. 1152-1163, 2012.

[13] S. Li and X. Meng, "Four edge-grafting theorems on the reciprocal degree distance of graphs and their applications," Journal of Combinatorial Optimization, vol. 30, no. 3, pp. $468-488,2015$.

[14] G.-X. Cai, X.-X. Li, and G.-D. Yu, "Maximum reciprocal degree resistance distance index of unicyclic graphs," Discrete Dynamics in Nature and Society, vol. 2020, pp. 1-14, 2020. 